\title{
Degeneracies in sky localization determination from a spinning coalescing binary through gravitational wave observations: a Markov-chain Monte Carlo analysis for two detectors
}

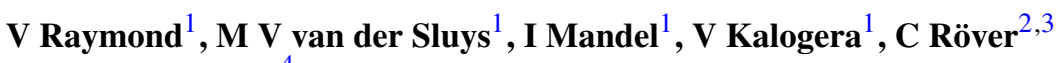 \\ and $\mathrm{N}$ Christensen ${ }^{4}$ \\ ${ }^{1}$ Department of Physics and Astronomy, Northwestern University, 2131 Tech Drive, Evanston, \\ IL 60208, USA \\ 2 Department of Statistics, University of Auckland, Private Bag 92019, Auckland 1142, \\ New Zealand \\ ${ }^{3}$ Max-Planck-Institut für Gravitationsphysik, Callinstraße 38, 30167 Hannover, Germany \\ ${ }^{4}$ Physics and Astronomy Department, Carleton College, One North College Street, Northfield \\ MN 55057, USA \\ E-mail: vivien@u.northwestern.edu
}

Received 20 December 2008, in final form 4 March 2009

Published 19 May 2009

Online at stacks.iop.org/CQG/26/114007

\begin{abstract}
Gravitational-wave signals from inspirals of binary compact objects (black holes and neutron stars) are primary targets of the ongoing searches by groundbased gravitational-wave interferometers (LIGO, Virgo and GEO-600). We present parameter-estimation simulations for inspirals of black-hole-neutronstar binaries using Markov-chain Monte Carlo methods. As a specific example of the power of these methods, we consider source localization in the sky and analyze the degeneracy in it when data from only two detectors are used. We focus on the effect that the black-hole spin has on the localization estimation. We also report on a comparative Markov-chain Monte Carlo analysis with two different waveform families, at 1.5 and 3.5 post-Newtonian orders.
\end{abstract}

PACS numbers: $02.50 .-\mathrm{r}, 02.70 . \mathrm{Uu}, 04.30 . \mathrm{Tv}, 04.80 . \mathrm{Nn}$, 95.85.Sz

(Some figures in this article are in colour only in the electronic version)

\section{Introduction}

Binary systems with compact objects—-neutron stars (NSs) and black holes (BHs) - in the mass range $\sim 1 M_{\odot}-100 M_{\odot}$ are among the most likely sources of gravitational waves (GWs) for ground-based laser interferometers currently in operation (Cutler and Thorne 2002): LIGO 
(Barish and Weiss 1999), Virgo (Acernese et al 2004) and GEO-600 (Willke et al 2004). Merger-rate estimates are quite uncertain and for BH-NS binaries current detection-rate estimates range from 0.0003 to $0.1 \mathrm{yr}^{-1}$ for first-generation instruments (e.g. O'Shaughnessy et al (2008)). Upgrades to enhanced LIGO/Virgo (2008-2009) and advanced LIGO/Virgo (2011-2014) are expected to increase detection rates by factors of about $\sim 10$ and $\sim 10^{3}$, respectively.

The measurement of source properties holds major promise for improving our astrophysical understanding and requires reliable methods for parameter estimation. This is a challenging problem, however, because of the large number of parameters ( 9 for circular non-spinning binaries and more for spinning systems) and the significant amount of structure in the parameter space. In the case of low-mass-ratio binaries (e.g. BH-NS), these issues are amplified for significant spin magnitudes and large misalignments between the $\mathrm{BH}$ spin and the orbital angular momentum (Apostolatos et al 1994, Grandclément et al 2003, Buonanno et al 2003). However, the presence of spins improves parameter estimation through the signal modulations, although still presenting us with a considerable computational challenge. This was highlighted in the context of LISA observations (see Vecchio (2004), Lang and Hughes (2006)) and in our first study devoted to ground-based observations (van der Sluys et al 2008b).

In this paper we examine the potential for parameter estimation of spinning binary inspirals with ground-based interferometers. Röver et al (2006, 2007a) explored parameter estimation for non-spinning binaries, which requires nine parameters. We focus on BH-NS binaries, which can exhibit significant coupling between the orbital angular momentum and the $\mathrm{BH}$ spin, mainly because of the high-mass ratio (Apostolatos et al 1994), while at the same time we are justified to ignore the NS spin, leading to a 12-dimensional parameter space. We apply a newly developed Markov-chain Monte Carlo (MCMC) algorithm (van der Sluys et al 2008a) to spinning inspiral signals injected into synthetic noise and we derive posterior probability density functions (PDFs) of all 12 signal parameters. In our previous study (van der Sluys et al 2008b), we showed the accuracy obtained in sky-position determination using data from a two-detector network, where a degeneracy in the sky position exists, and from a three-detector network, where the degeneracy is broken. Following this work, we here analyze in further detail the degeneracy which is present when data from only two detectors are used. In section 4.2, we show that the degeneracy in the sky position is reduced but not lifted when a significant spin is present $\left(a_{\text {spin }} \geqslant 0.5\right)$ and that a sufficient angle between the spin and orbital angular momentum can break such a degeneracy $\left(\theta_{S L} \geqslant 55^{\circ}\right)$. In this study, we demonstrate that these degeneracies are due to a high degree of similarity between signals from sources with significantly different parameter sets, while we made sure that the observed effects are real and not in fact artifacts due to potential errors in our analysis methodology.

In section 4.3 , we also demonstrate that the inclusion of higher post-Newtonian orders in the waveform can improve the accuracy of intrinsic-parameter estimation. Meanwhile, we find that the additional structure in the parameter space of higher order waveforms lowers the sampling efficiency of the MCMC and requires improvements to the sampling scheme.

\section{Signal and observables}

In this paper we analyze the signal produced during the inspiral phase of two compact objects of masses $M_{1,2}$ in a circular orbit. We focus on a fiducial BH-NS binary system with $M_{1}=10 M_{\odot}$ and $M_{2}=1.4 M_{\odot}$, and we ignore the NS spin. The BH spin $\mathbf{S}$ couples to the orbital angular momentum $\mathbf{L}$, leading to amplitude and phase modulations of the observed gravitational radiation due to the precession of the orbital plane. Here we model GWs by 
post-Newtonian $(\mathrm{pN})$ waveforms, either at $1.5 \mathrm{pN}$ order or at $3.5 \mathrm{pN}$ order in phase and Newtonian amplitudes. For the latter waveform we use the implementation from the LSC Algorithm Library (LAL) (LIGO Scientific Collaboration 2007). In our analysis we model the noise in each detector as a zero-mean Gaussian, stationary random process, with one-sided noise spectral density $S_{a}(f)$, at the initial-LIGO design sensitivity (Fritschel 2003).

\subsection{Waveform template at the $1.5 \mathrm{pN}$ order}

We adopt the simple-precession limit (equations (51), (52), (59) and (63) in Apostolatos et al (1994)), appropriate for the single-spin system considered here. For simplicity (to speed up the waveform calculation), we also ignore the Thomas precession (Apostolatos et al 1994). In this approximation, the orbital angular momentum $\mathbf{L}$ and spin $\mathbf{S}$ precess with the same angular frequency around a fixed direction $\hat{\mathbf{J}}_{0} \approx \hat{\mathbf{J}}$, where $\mathbf{J}=\mathbf{L}+\mathbf{S}$. During the inspiral phase the spin misalignment $\theta_{S L} \equiv \arccos (\hat{\mathbf{S}} \cdot \hat{\mathbf{L}})$ and $S=|\mathbf{S}|$ are constant. These approximate waveforms retain (at the leading order) all the prominent qualitative features introduced by the spins, while allowing us to rapidly compute the waveforms analytically. While this approach is justified for the exploration of GW astronomy and the development of parameter-estimation algorithms, more accurate waveforms (e.g. Kidder (1995), Will and Wiseman (1996), Faye et al (2006), Blanchet et al (2006)) will be necessary for the analysis of real signals (see section 4.3).

A circular binary inspiral with one spinning compact object is described by a 12 dimensional parameter vector $\vec{\lambda}$. With respect to a fixed geocentric coordinate system, our choice of independent parameters is

$$
\vec{\lambda}=\left\{\mathcal{M}, \eta, \mathrm{RA}, \sin \text { Dec, } \sin \theta_{J_{0}}, \phi_{J_{0}}, \log d_{\mathrm{L}}, a_{\mathrm{spin}}, \cos \theta_{S L}, \phi_{\mathrm{c}}, \alpha_{\mathrm{c}}, t_{\mathrm{c}}\right\},
$$

where $\mathcal{M}=\frac{\left(M_{1} M_{2}\right)^{3 / 5}}{\left(M_{1}+M_{2}\right)^{1 / 5}}$ and $\eta=\frac{M_{1} M_{2}}{\left(M_{1}+M_{2}\right)^{2}}$ are the chirp mass and symmetric mass ratio, respectively, RA (right ascension) and Dec (declination) identify the source position in the sky, the angles $\theta_{J_{0}} \in\left[-\frac{\pi}{2}, \frac{\pi}{2}\right]$ and $\phi_{J_{0}} \in\left[0,2 \pi\right.$ [ identify the unit vector $\hat{\mathbf{J}}_{0}, d_{\mathrm{L}}$ is the luminosity distance to the source and $0 \leqslant a_{\text {spin }} \equiv S / M_{1}^{2} \leqslant 1$ is the dimensionless spin magnitude, $\phi_{\mathrm{c}}$ and $\alpha_{\mathrm{c}}$ are integration constants that specify the GW phase and the location of $\mathbf{S}$ on the precession cone, respectively, at the time of coalescence $t_{\mathrm{c}}$, defined with respect to the center of the Earth.

Given a network comprising $n_{\text {det }}$ detectors, the data collected at the $a$ th instrument $\left(a=1, \ldots, n_{\text {det }}\right)$ is given by $x_{a}(t)=n_{a}(t)+h_{a}(t ; \vec{\lambda})$, where $h_{a}(t ; \vec{\lambda})=F_{a,+}(t) h_{a,+}(t ; \vec{\lambda})+$ $F_{a, \times}(t) h_{a, \times}(t ; \vec{\lambda})$ is the GW strain at the detector (see equations (2)-(5) in Apostolatos et al (1994)) and $n_{a}(t)$ is the detector noise. The astrophysical signal is given by the linear combination of the two independent polarizations $h_{a,+}(t ; \vec{\lambda})$ and $h_{a, \times}(t ; \vec{\lambda})$ weighted by the time-dependent antenna beam patterns $F_{a,+}(t)$ and $F_{a, \times}(t)$, respectively.

\subsection{Waveform template in the $3.5 \mathrm{pN}$ order}

Although the $1.5 \mathrm{pN}$, simple-precession waveform is useful to investigate the principal effects of spin on parameter estimation, a more realistic waveform is needed to analyze detected signals. The waveform we use for this is $3.5 \mathrm{pN}$ in phase and Newtonian amplitude. The implementation in the LSC Algorithm Library closely follows the first section of (Buonanno et al 2003). For comparison purposes we converted the usual set of parameters used in the LAL software to the parameters in equation (1). In doing so, we fix 3 of the 15 parameters of the LAL parameter set, setting the spin of the second member of the binary to be 0 . The waveform is generated using LALGenerateInspiral () from the injection package (LIGO Scientific Collaboration 2007). An example of $h_{a}(t)$ for $a_{\text {spin }}=0.5$ and $\theta_{S L}=20^{\circ}$ for both waveforms is shown in figure 1. 


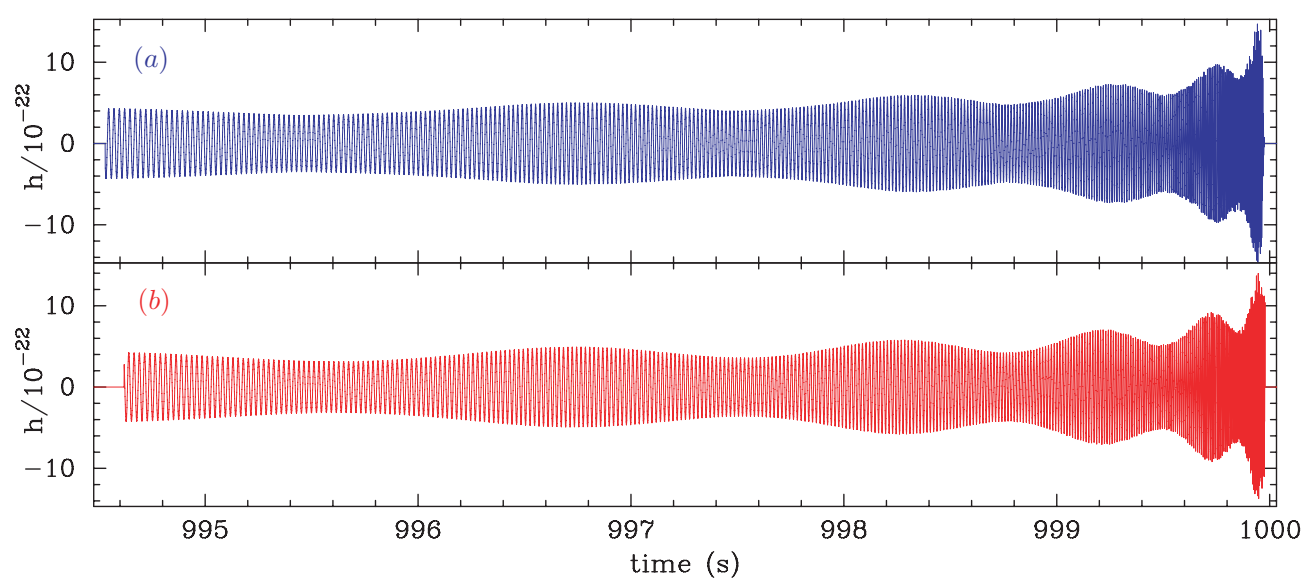

Figure 1. (a) Part of the $1.5 \mathrm{pN}$ time-domain waveform from a source with $a_{\text {spin }}=0.5$ and $\theta_{S L}=20^{\circ}$. (b) The $3.5 \mathrm{pN}$ waveform from a source with the same parameters. The waveforms start at $40 \mathrm{~Hz}$ and are aligned at the coalescence time.

\section{Parameter estimation: methods}

The goal of our analysis is to determine the posterior PDF of the unknown parameter vector $\vec{\lambda}$ in equation (1), given the data sets $x_{a}$ collected by a network of $n_{\text {det }}$ detectors and the prior $p(\vec{\lambda})$ on the parameters. We use wide, flat priors (see van der Sluys et al (2008a) for details). The Bayes theorem provides a rigorous mathematical rule to assign such a probability:

$$
p\left(\vec{\lambda} \mid x_{a}\right)=\frac{p(\vec{\lambda}) \mathcal{L}\left(x_{a} \mid \vec{\lambda}\right)}{p\left(x_{a}\right)}
$$

in the previous equation

$$
\mathcal{L}\left(x_{a} \mid \vec{\lambda}\right) \propto \exp \left(\left\langle x_{a} \mid h_{a}(\vec{\lambda})\right\rangle-\frac{1}{2}\left\langle h_{a}(\vec{\lambda}) \mid h_{a}(\vec{\lambda})\right\rangle\right)
$$

is the likelihood function of the data given in the model, which measures the fit of the data to the model, and $p\left(x_{a}\right)$ is the marginal likelihood or evidence. We use the notation

$$
\langle x \mid y\rangle=4 \operatorname{Re}\left(\int_{f_{\text {low }}}^{f_{\text {high }}} \frac{\tilde{x}(f) \tilde{y}^{*}(f)}{S_{a}(f)} \mathrm{d} f\right)
$$

to denote the overlap of signals $x$ and $y$, where $\tilde{x}(f)$ is the Fourier transform of $x(t)$. For future reference, we also define a match between two waveforms corresponding to different parameter values as the overlap between the normalized waveforms:

$$
M\left(h\left(\vec{\lambda}_{1}\right), h\left(\vec{\lambda}_{2}\right)\right)=\frac{\left\langle h\left(\vec{\lambda}_{1}\right) \mid h\left(\vec{\lambda}_{2}\right)\right\rangle}{\sqrt{\left\langle h\left(\vec{\lambda}_{1}\right) \mid h\left(\vec{\lambda}_{1}\right)\right\rangle\left\langle h\left(\vec{\lambda}_{2}\right) \mid h\left(\vec{\lambda}_{2}\right)\right\rangle}} .
$$

Note that with the definitions employed here, the 'true' likelihood computed at the parameters of the injected waveform $\mathcal{L}_{\text {true }}\left(x \mid \vec{\lambda}_{\text {true }}\right)$ is a random variable that depends on a particular realization of the noise $n_{a}$ in the data $x_{a}=h\left(\vec{\lambda}_{\text {true }}\right)+n_{a}$. We define the signal-to-noise ratio (SNR) to be the square root of twice the expectation value of $\log \mathcal{L}_{\text {true }}$ :

$$
\mathrm{SNR}=\sqrt{\left\langle h\left(\vec{\lambda}_{\text {true }}\right) \mid h\left(\vec{\lambda}_{\text {true }}\right)\right\rangle} .
$$


For multi-detector observations involving a network of detectors with uncorrelated noisethis is the case of this paper, where we use up to two non-collocated detectors-we have $\mathcal{L}(\vec{x} \mid \vec{\lambda})=\prod_{a=1}^{n_{\mathrm{det}}} \mathcal{L}\left(x_{a} \mid \vec{\lambda}\right)$, for $\vec{x} \equiv\left\{x_{a}: a=1, \ldots, n_{\mathrm{det}}\right\}$ and

$$
p(\vec{\lambda} \mid \vec{x})=\frac{p(\vec{\lambda}) \mathcal{L}(\vec{x} \mid \vec{\lambda})}{p(\vec{x})} .
$$

The numerical computation of the joint and marginalized PDFs involves the evaluation of integrals over a large number of dimensions. MCMC methods (e.g. Gilks et al (1996), Gelman et al (1997) and references therein) have proved to be especially effective in tackling these numerical problems. We developed an adaptive (see Figueiredo and Jain (2002), Atchadé and Rosenthal (2005)) MCMC algorithm to explore the parameter space efficiently while requiring the least amount of tuning for the specific signal at hand; the code is an extension of the one developed by some of the authors to explore MCMC methods for non-spinning binaries (Röver et al 2006, 2007a) and takes advantage of techniques explored by some of us in the context of LISA data analysis (Stroeer et al 2007). A summary of the methods used in our MCMC code was published in van der Sluys et al (2008a); more technical details will be provided elsewhere.

\section{Parameter estimation: results}

\subsection{MCMC runs}

Here we present results obtained by injecting a signal into simulated interferometer noise and computing the posterior PDFs with MCMC techniques, for a fiducial source consisting of a $10 M_{\odot}$ spinning $\mathrm{BH}$ and a $1.4 M_{\odot}$ non-spinning NS in a binary system at a distance of about $16 \mathrm{Mpc}$ (see sections 4.2 and 4.3 for parameters values). We consider a number of cases for which we change the $\mathrm{BH}$ spin parameters. We run the analysis using the simulated data from (i) the $4 \mathrm{~km}$ LIGO detector at Hanford (H1) alone and (ii) the LIGO Hanford (H1) and a second detector with the initial-LIGO noise curve located and oriented in the same way as the Virgo detector near Pisa, which we denote by $(\mathrm{P})$.

The MCMC analysis that we carry out on each data set consists of 5-25 independent chains, each with a length of several million iterations. The chains are sampled after a burn-in period (see e.g. Gilks et al (1996)) that is determined automatically as follows: we determine the absolute maximum likelihood $\log \left(\mathcal{L}_{\text {max }}\right)$, defined as the highest likelihood $\log [\mathcal{L}(\vec{x} \mid \vec{\lambda})]$ obtained over the ensemble of parameters $\vec{\lambda}$ for which the overlap has been computed, for any of the individual chains. Then for each chain we include all the iterations after the chain reaches a likelihood value of $\log \left(\mathcal{L}_{\max }\right)-2$ for the first time. All our Markov chains start at offset (i.e., non-true) parameter values, unless specified otherwise. The starting values for $\mathcal{M}$ and $t_{\mathrm{c}}$ are drawn from a Gaussian distribution centered on the true parameter value with a standard deviation of $0.1 M_{\odot}$ and $30 \mathrm{~ms}$, respectively. The other ten parameters are drawn uniformly from the allowed ranges. Our MCMC code needs to run from typically a few days to one week in order to show the first results and 10-14 days to accumulate a sufficient number of iterations for good statistics, each chain using a single $2.8 \mathrm{GHz} \mathrm{CPU}$.

\subsection{A ring in the sky}

For the study in this section, we use the $1.5 \mathrm{pN}$ waveform with the simple-precession prescription only (see section 2.1). In order to further speed up the MCMC runs, we fixed the mass and spin parameters to the true parameter values and performed test calculations to verify that this does not affect our conclusions. 
Table 1. List of the experiments described in section 4.2. The sky ring is defined as the ring produced by experiment 1 , composed of arcs and gaps.

\begin{tabular}{llll}
\hline Experiment & $\begin{array}{l}\text { Injection position } \\
\text { (in the sky ring) }\end{array}$ & $\begin{array}{l}\text { Sky position } \\
\text { parameters }\end{array}$ & $\begin{array}{l}\text { Starting values } \\
\text { (in the sky ring) }\end{array}$ \\
\hline 1 & Arc & Free & Offset (non-true values) \\
$2(a)$ & Arc & Fixed & True position (arc) \\
$2(b)$ & Arc & Fixed & Gap position $(O)$ \\
3 & Gap $(O)$ & Free & Offset (non-true values)
\end{tabular}

As reference MCMC runs (experiment 1, see table 1), we injected signals into two simulated detectors ( $\mathrm{H} 1$ and $\mathrm{P})$. We made three different injections, which differed in the spin magnitude values $\left(a_{\text {spin }}=0.0,0.5\right)$ and the spin-misalignment values $\left(\theta_{S L}=20^{\circ}, 55^{\circ}\right)$ for the $a_{\text {spin }}=0.5$ case; the remaining parameters were kept constant across all three injections: $\mathcal{M}=3.0 M_{\odot}, \eta=0.11\left(M_{1}=10.0 M_{\odot}, M_{2}=1.4 M_{\odot}\right), d_{\mathrm{L}}=16 \mathrm{Mpc}, \mathrm{R} . \mathrm{A} .=14.3 \mathrm{~h}$, Dec $=$ $11.5^{\circ}, \theta_{J_{0}}=3.8^{\circ}, \phi_{J_{0}}=289^{\circ}, \phi_{\mathrm{c}}=305^{\circ}, \alpha_{\mathrm{c}}=4^{\circ}$ and $t_{\mathrm{c}}=700009012.345 \mathrm{~s}$ GPS time.

For a detection with two interferometers, the sky position is degenerate; when no spin is present in the source, our PDFs show an incomplete sky ring where the source might be long arcs separated by empty 'gaps' rather than one tightly constrained ring. When the $\mathrm{BH}$ is spinning, and especially when the misalignment angle between orbital angular momentum and spin is significant, the sky location constraint shrinks appreciably until much smaller arcs-or even a single arc-are left (see figure $2(a)$ ).

In order to probe the nature of the gaps in the sky ring, we devised a second experiment (experiment 2, see table 1). For each run, we injected the same signal as before, but now forced the MCMC code to search for the other parameters while keeping the sky position fixed to either the true values (experiment $2(a)$ ) or to the sky position in the gap that is labeled by the circle in the first panel of figure 2(a) (experiment $2(b)$ ). Running the MCMC code in this way provides us with the combination of the free parameters that fits the data best, given the constraint in the sky position (the conditional posterior distribution, conditional on the corresponding sky position). In particular, the code provides us with the highest likelihood that can be obtained for this sky position. These likelihoods are listed in table 2. They show that the maximum likelihood found in the gap is very similar to the likelihood of the injection for the non-spinning signal, whereas it is significantly lower in the case of the spinning signal. For the non-spinning signal, an unfavorable binary orientation (inclination $=92.0^{\circ}$ ) and hence a short distance $\left(d_{\mathrm{L}}=3.6 \mathrm{Mpc}\right)$ are needed to give a good match for the given sky position. The modulations of the signal due to the precession of the binary orbit prevent this match in the spinning cases.

Thus, for a source with a non-spinning $\mathrm{BH}$, a high likelihood can be found in the gaps of the sky ring. Therefore, the absence of high likelihood values does not explain the fact that our Markov chains hardly sample this part of the parameter space. Instead, we find that the PDFs for some of the other parameters (especially the distance $d_{\mathrm{L}}$ and binary orientation $\arccos (\mathbf{J} \cdot \mathbf{N}))$ are very narrow for experiment $2(b)$ compared to those for experiment $2(a)$ (figure 3). This indicates that the overall volume of parameter space in these parameters is very small for the sky positions in the gap and this is the reason that these gaps are not visited frequently by the chains. On a side note, our priors are flat and cannot be the cause for this effect. If our MCMC runs had infinite length, they would sample this region in a parameter space and bridge the gaps in figure $2(a)$ with a thin line, i.e. the points 'bridging' the gaps will form a set of very small measure. Even with a perfect sampling, the same behavior is 
(a)
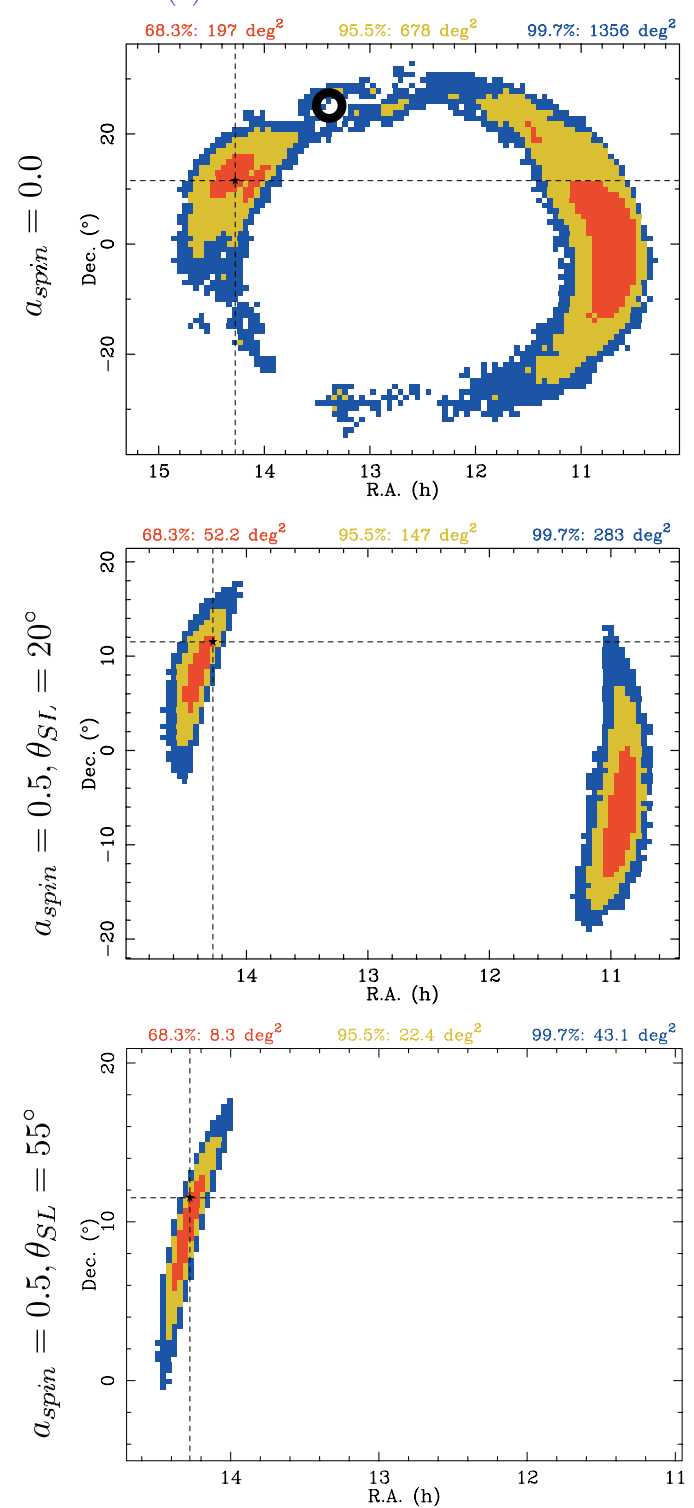

(b)
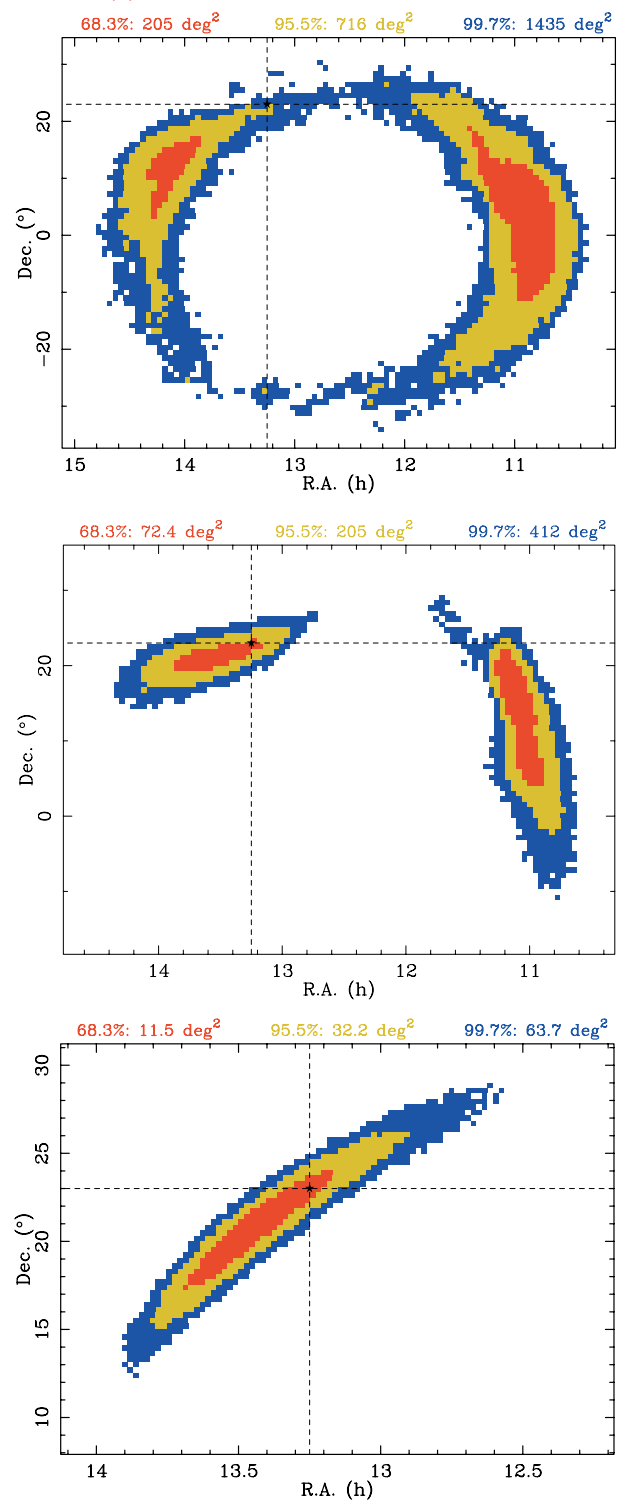

Figure 2. Two-dimensional PDFs of the sky position for the MCMC runs as labeled. The colors show the different probability intervals ( $1 \sigma, 2 \sigma$ and $3 \sigma$ for red (center), yellow (middle) and blue (periphery), respectively). The black dashed lines mark the position in the sky of the injection for each run. (a) Results for the reference runs, experiment 1 (signal injection at RA $=14.3 \mathrm{~h}$, Dec $=11.5^{\circ}$ ). The symbol $\bigcirc$ denotes the 'gap' discussed in the text. $(b)$ Results for experiment 3: an MCMC run with a signal injection at $\mathrm{O}\left(\mathrm{RA}=13.25 \mathrm{~h}, \mathrm{Dec}=23^{\circ}\right)$. For the non-spinning case, the PDFs are very similar to those in the original run, whereas they are very different for the spinning cases (note the difference in the axis ranges).

to be expected: the chains would mainly sample the arcs and rarely the gaps. Interestingly, the similarity in likelihood values between the true position and the gap for the case of a non-spinning $\mathrm{BH}$ also means that these two signals are virtually indistinguishable, i.e. their 
Table 2. Likelihood values recovered by the MCMC runs of experiment $2(b)$ for the sky ring, described in section 4.2 .

\begin{tabular}{|c|c|c|c|c|}
\hline & $a_{\text {spin }}=0.0$ & $\begin{array}{l}a_{\text {spin }}=0.5 \\
\theta_{S L}=20^{\circ}\end{array}$ & $\begin{array}{l}a_{\text {spin }}=0.5 \\
\theta_{S L}=55^{\circ}\end{array}$ & \\
\hline Network SNR & 17.0 & 18.3 & 18.4 & \\
\hline $\log \left(\mathcal{L}\left(x_{a} \mid \vec{\lambda}_{\text {true }}\right)\right)$ & 131 & 154 & 208 & \\
\hline $\log \left(\mathcal{L}_{\max }\right)(\bigcirc)$ & 131 & 125 & 152 & \\
\hline $\begin{array}{l}\text { Match } M\left(h\left(\vec{\lambda}_{\text {true }}\right), h\left(\vec{\lambda}_{\mathcal{L}_{\text {max }}}\right)\right) \\
\text { (equation }(5))\end{array}$ & $99.5 \%$ & $89.6 \%$ & $82.5 \%$ & $\begin{array}{l}\text { (between waveforms } \\
\text { injected in experiment } \\
1 \text { and those } \\
\text { corresponding to } \mathcal{L}_{\text {max }} \text { ) }\end{array}$ \\
\hline $\mathcal{L}_{\text {max }}$ parameters : & & & & $\begin{array}{l}\text { (reference parameters, } \\
\text { injected in } \\
\text { experiment } 1 \text { ) }\end{array}$ \\
\hline Position (RA [h], Dec. $\left.\left[{ }^{\circ}\right]\right)$ & $13.25,23$ & $13.25,23$ & $13.25,23$ & $14.3,11.5$ \\
\hline Orientation $\left(\theta_{J_{0}}\left[{ }^{\circ}\right], \phi_{J_{0}}\left[^{\circ}\right]\right)$ & $-65.4,10.8$ & $-59.5,340.4$ & $-21.8,169.5$ & $3.8,289$ \\
\hline Inclination $(\arccos (\mathbf{J} \cdot \mathbf{N}))\left[^{\circ}\right]$ & 92.0 & 97.5 & 129.7 & 128.4 \\
\hline Distance [Mpc] & 3.7 & 11.1 & 18.3 & 16 \\
\hline$t_{\mathrm{c}}-700009010[\mathrm{~s}]$ & 2.34955 & 2.34959 & 2.34960 & 2.34500 \\
\hline
\end{tabular}

match (equation (5)) is very high $(99.5 \%$, see table 2$)$. This indicates that if the source were truly in the gap, Markov chains of this length would not recover it, and chains of any length would not have a significant PDF in the gaps.

To illustrate this, we did a third experiment (experiment 3, see table 1). We injected a signal at the position in the gap labeled by $O$ in figure $2(a)$. We kept the same masses and spin values as before and set the other parameters to the values yielding the maximum likelihoods from the second experiment, listed in table 2.

In the non-spinning case, the sky ring that is recovered is very similar to that of the original run (see figure $2(b)$ ); the small difference seen can be explained by the fact that the match between the signal injected in experiment 3 and the signal injected in experiment 1 was $99.5 \%$ and not $100 \%$ (table 2). This shows that there exist carefully selected combinations of the sky position, binary orientation and distance which cannot be easily recovered by our analysis. However, this reflects a real phenomenon; it is very improbable for a source to have the right orientation to produce the gravitational-wave signal we injected and have its sky location in the gap. And by giving preference to more likely solutions the parameter estimation routine is in fact doing the right, completely reasonable thing. The PDFs of the relevant parameters for the comparison of experiments 1 and 3 are plotted in figure 4. The PDFs in the other parameters are also very similar in both the original run and the third experiment run.

When the BH spins moderately, the two signals injected in experiment 1 and in experiment 3 are different (as we can infer from the large difference in likelihood in experiment $2(b)$ and the smaller match, see table 2). The different injections yield significantly different PDFs for the sky position (figure $2(b)$ ) as well as for the other parameters, as we checked in our study.

\subsection{Comparison of MCMC runs with $1.5 \mathrm{pN}$ and $3.5 \mathrm{pN}$ waveforms}

So far we have used $1.5 \mathrm{pN}$ waveforms for computational efficiency. However, in this section we used our MCMC implementation to present the first comparison of the accuracy 

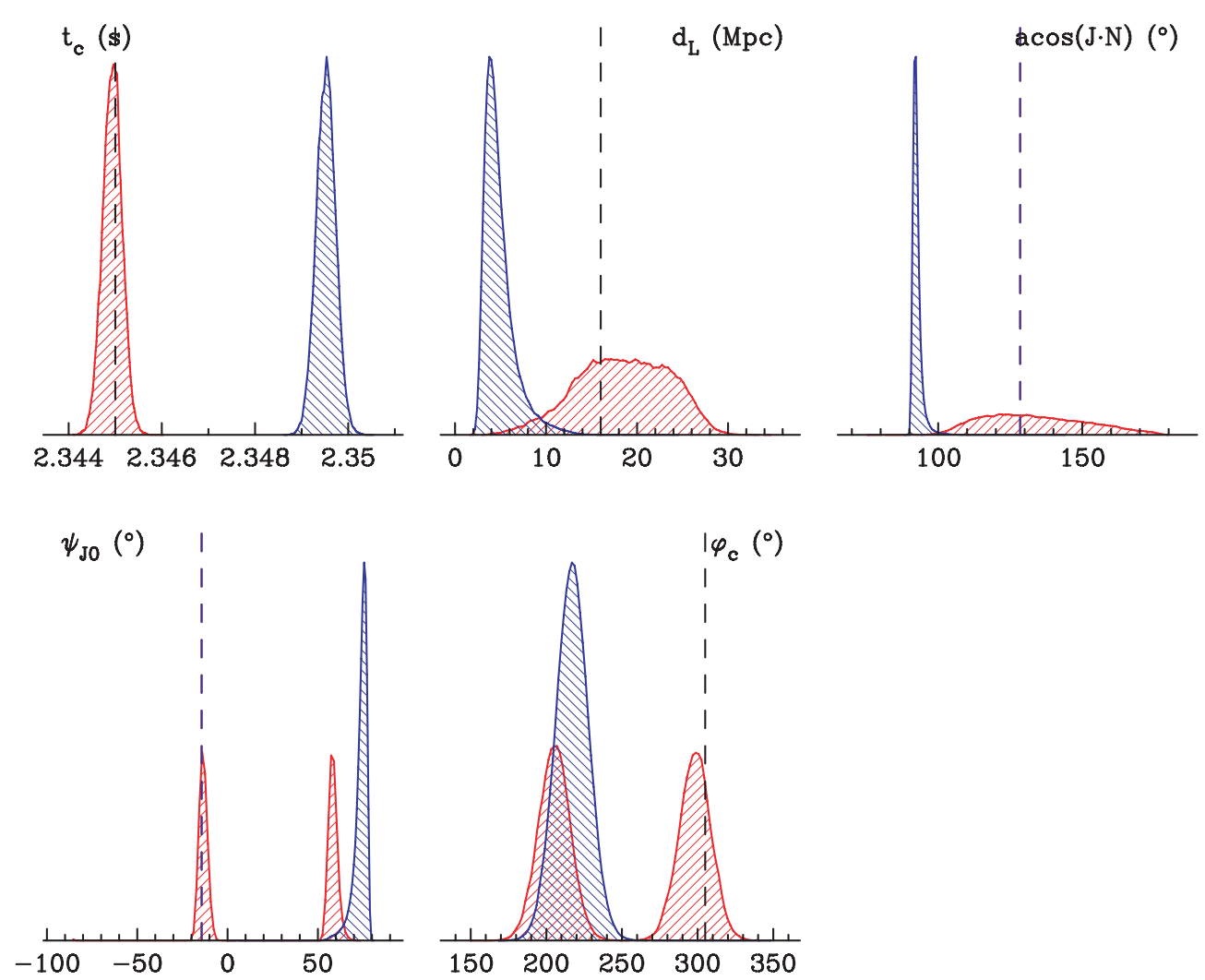

Figure 3. One-dimensional PDFs for the runs of experiment 2(a) (red, hatched upward) and of the runs in experiment $2(b)$ (blue, hatched downward) in the non-spinning case. The black dashed lines mark the values of the injected parameters. The level of support is indeed smaller in the second case.

of parameter estimation with the $1.5 \mathrm{pN}$ and $3.5 \mathrm{pN}$ waveform families, as described in section 2. In both cases, we injected BH-NS binary inspiral waveforms with a non-spinning $\mathrm{BH}$ into the noise of a single interferometer $(\mathrm{H} 1)$. We analyzed the $1.5 \mathrm{pN}$ injected waveform with an MCMC with $1.5 \mathrm{pN}$ waveform templates and the $3.5 \mathrm{pN}$ injected waveform with $3.5 \mathrm{pN}$ waveform templates. We scaled the distance to the source to obtain a signal-to-noise ratio of 20.0 in both cases, which resulted in a distance of $\sim 11.9 \mathrm{Mpc}$ for the $1.5 \mathrm{pN}$ waveform case and $\sim 12.2 \mathrm{Mpc}$ for the $3.5 \mathrm{pN}$ waveform case. The remaining injection parameters were set to $a_{\text {spin }}=0.0, \mathcal{M}=3.0 M_{\odot}, \eta=0.11\left(M_{1}=10.0 M_{\odot}, M_{2}=1.4 M_{\odot}\right), \mathrm{RA}=17.3 \mathrm{~h}, \mathrm{Dec}=$ $-5^{\circ}, \theta_{J_{0}}=-23^{\circ}, \phi_{J_{0}}=194^{\circ}, \phi_{\mathrm{c}}=352^{\circ}$ and $t_{\mathrm{c}}=894377000.500244 \mathrm{~s}$ for this study. The three spin parameters $\left(a_{\text {spin }}, \theta_{S L}\right.$ and $\left.\alpha_{\mathrm{c}}\right)$ were fixed, forcing the chains to explore a parameter space that was effectively nine dimensional.

Figure 5 compares the PDFs of the mass parameters for the runs with the $1.5 \mathrm{pN}$ and $3.5 \mathrm{pN}$ waveforms for $1.5 \times 10^{6}$ iterations in both cases. It is evident that the estimation of the symmetric mass ratio $\eta$ is more accurate in the $3.5 \mathrm{pN}$ case, which results in better constraints on the individual masses. The $2 \sigma$ probability ranges for the chirp mass are roughly similar in both cases (a factor of 1.2 narrower when the $3.5 \mathrm{pN}$ waveform is used), whereas for $\eta$, and hence for the individual masses, the ranges are narrower by a factor of 1.8 when the $3.5 \mathrm{pN}$ 

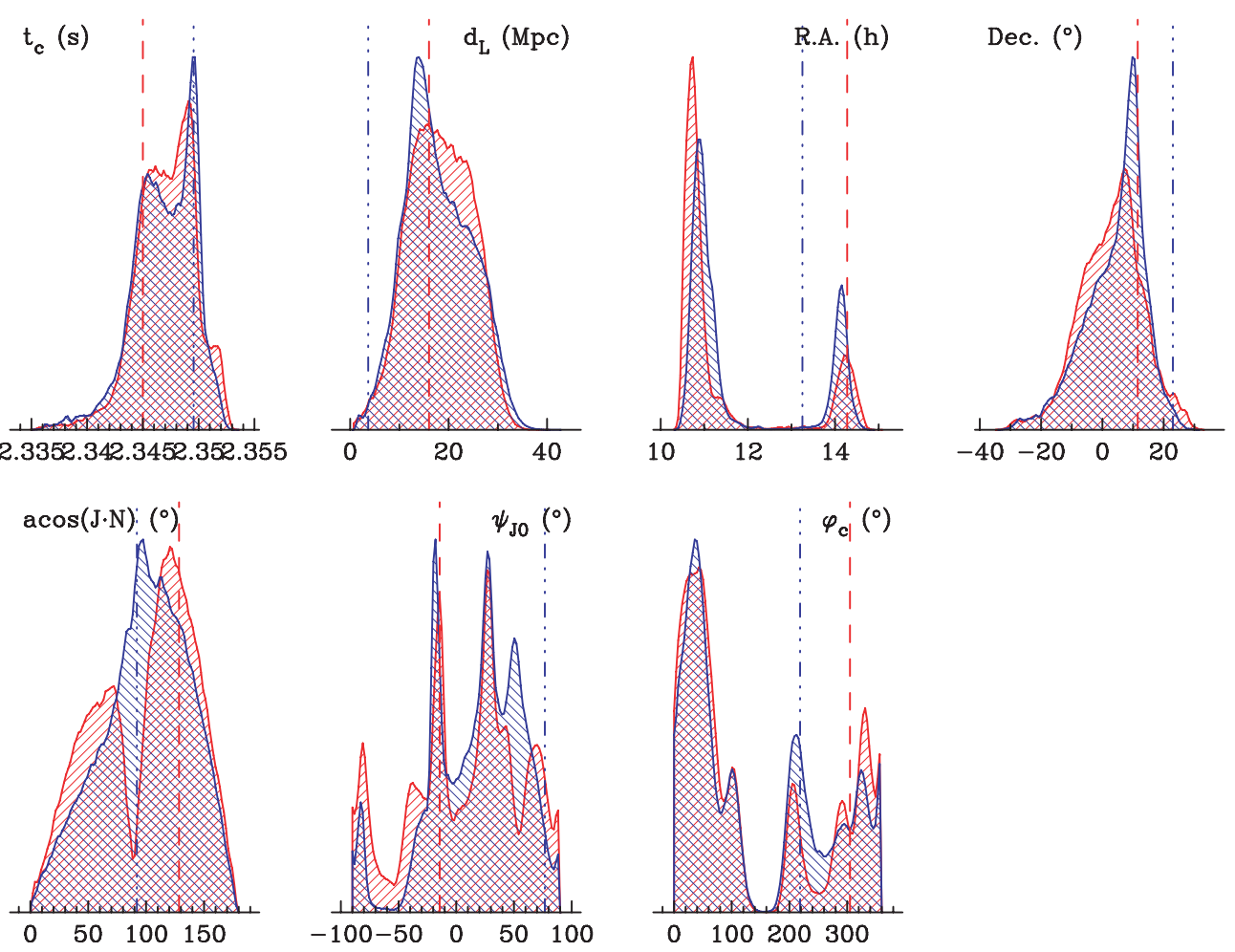

Figure 4. One-dimensional PDFs for the reference run in experiment 1 (red, hatched upward) and of the runs in experiment 3 (blue, hatched downward) in the non-spinning case. The dashed lines mark the values of the injected parameters in experiment 1 , while the dotted-dashed lines mark the values of the injected parameters in experiment 3. The PDFs for all parameters are very similar for the two injections.

waveform is used. The additional information in the higher order $\mathrm{pN}$ waveforms results in a greater structure of the parameter space. In principle, this extra structure allows us to estimate the binary parameters more accurately. However, a more structured parameter space also affects the sampling efficiency of our MCMC code negatively, so that we need more iterations to obtain a well-sampled MCMC run. In addition, the computation of a single $3.5 \mathrm{pN}$ waveform template takes about 2.4 times longer than that of a $1.5 \mathrm{pN}$ template. This effect prevents us, for now, from performing this analysis in a full 12-dimensional or 15-dimensional parameter space in a reasonably short computational time.

One of the ways to speed up the convergence of the code is to use faster, lower order $\mathrm{pN}$ waveform templates during the burn-in phase and to use the additional information provided by the more expensive, higher order $\mathrm{pN}$ waveforms to sample the true mode of the PDF accurately. In addition, we have plans to use time annealing (Gair et al 2008), starting the MCMC runs on only a fraction of the available time-domain data, so that only a part of the waveform template needs to be computed for each iteration. These improvements will speed up the computation of each waveform template and the loss of information will allow the Markov chains to move through the parameter space more easily until they lock on to the true modes of the PDF. If the amount of information is then gradually increased (by increasing the $\mathrm{pN}$ order of the waveforms or the time duration of the data set), the accuracy of parameter estimation will be improved. 

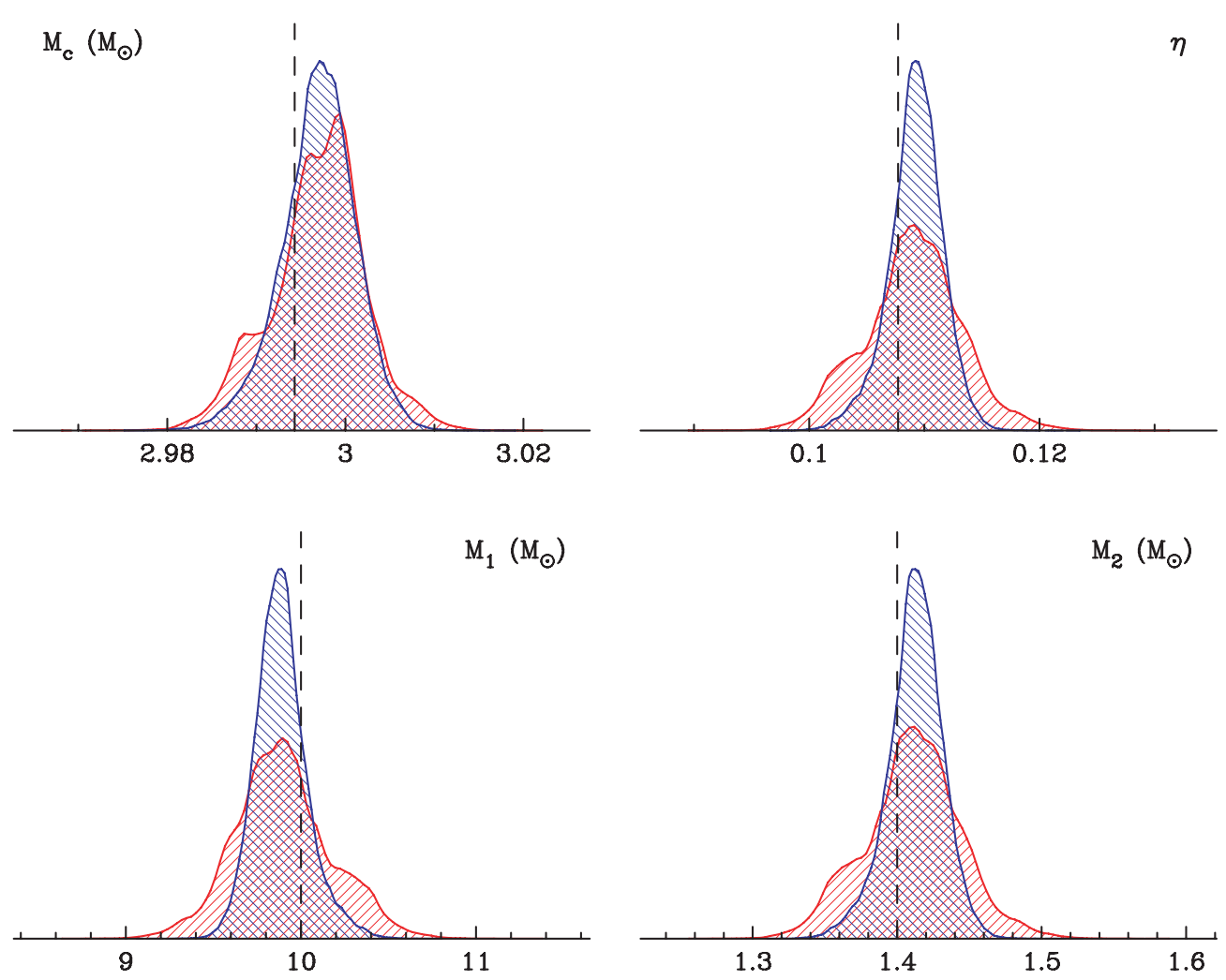

Figure 5. PDFs from a run with chains starting from the true values, with the $1.5 \mathrm{pN}$ waveform (red, hatched upward) and the $3.5 \mathrm{pN}$ waveform (blue, hatched downward) from a non spinning source. Only one detector (LIGO at Hanford) is used.

\section{Conclusions}

We have explored the degeneracies in the sky position for a gravitational-wave observation of an inspiral of a BH-NS binary with two non-collocated ground-based interferometers. Whereas simple triangulation based on time delays alone would result in a homogeneous sky ring, our MCMC runs show (in experiment 1) an incomplete ring in the sky consisting of arcs separated by gaps. While the arcs make up most of the circumference of the sky ring for an inspiral with a non-spinning $\mathrm{BH}$, these arcs become smaller when spin is present and may be reduced to a single arc for the case of moderate spin and a sufficient misalignment between the $\mathrm{BH}$ spin and the orbital angular momentum. We demonstrated that in the spinning case, the maximum likelihood values are in fact lower in the gaps than in the arcs. In the non-spinning case the likelihood in the gaps can actually be as high as in the arcs; however the gaps can be explained by a smaller volume of support in the other extrinsic parameters, such as the binary orientation and distance (experiment 2). It is then less likely for the chains to sample this smaller volume in the parameter space, resulting in gaps in the two-dimensional sky probability density function. In the non-spinning case, if a source is located in a gap, the posterior PDF still has a gap at the true source sky location (experiment 3).

The subject of estimating the position of a gravitational-wave source on the sky has been explored by many researchers, in the context of binary-inspiral and burst signals; the 
assumed baseline for these studies corresponds to three detectors located at the three LIGOVirgo observatory positions and operating at their design sensitivities. For detectable burst sources ( SNR > 5), arrival-time techniques should allow a precision of about $1^{\circ}$ for the source direction (Cavalier et al 2006). A method that takes into account burst-signal arrival time and amplitude, plus arrival-time uncertainties equivalent to what is observed with real LIGO and Virgo data, gives source uncertainties of a few degrees (Markowitz et al 2008). Coherent techniques also exist for burst detection and sky-location determination, but by their own admission these methods are computationally costly (Searle et al 2008). Many techniques have also been developed for sky localization for binary-inspiral events. It has been shown, using time and mass parameter estimates, that the LIGO-Virgo network could localize the position of a binary-neutron-star inspiral to an accuracy of $4^{\circ}$ if it were located in M87 $(16 \mathrm{Mpc})$ or $2^{\circ}$ for a source in NGC $6744(10 \mathrm{Mpc})$ (Beauville et al 2008); coherent methods, which also depend on estimating the time and mass parameters, give similar accuracies (Ajith and Bose 2009).

MCMC parameter-estimation methods, like those used in this study, are capable of estimating the sky parameters, along with all of the other signal parameters; this is one reason why MCMC methods are computationally intensive. Coherent MCMC methods applied to signals observed by the LIGO-Virgo network will be able to resolve the sky location to $2^{\circ}$ for signals with an SNR of 15 (Röver et al 2007b). When the compact objects have spin and the search templates account for this parameter, sky localization becomes relatively more accurate for higher values of spin (van der Sluys et al 2008b). It is important to remember that, in principle, the MCMC results show the best constraints one could hope to place on signal parameters (including the sky location) by displaying the true posterior PDFs. While comparisons with other particular sky-localization results may be cumbersome because different waveforms were assumed at different SNRs in different detectors, MCMC-produced posterior PDFs display the statistically correct and most precise localization. Bayesian methods achieve better parameter-estimation accuracy when the template model describes the functional form of the actual signal more accurately. When a gravitational-wave detection occurs, it is likely that all possible sky localization algorithms will be used, and the methods should be considered to be complementary.

In section 4.3, we compared MCMC results on software injections using waveform families of $1.5 \mathrm{pN}$ and $3.5 \mathrm{pN}$ orders for both the injections and the MCMC parameter estimation respectively. We have shown that the higher order templates have the potential for more accurate parameter estimation, but that sampling the parameter space with these templates is more computationally difficult. However, a number of upcoming improvements should improve the sampling efficiency of our MCMC runs.

The analysis presented here is the second step of a more detailed study that we are currently carrying out, exploring a much larger parameter space, developing techniques to reduce the computational cost of these simulations and testing the methods with actual LIGO data. We are in the process of updating our MCMC code to include the spin of the second binary member, increasing the dimensionality of the parameter space from 12 to 15 . Finally, we intend to further develop our Bayesian approach into a standard tool that can be included in the follow-up analysis pipeline used for the processing of the 'science data' collected by ground-based laser interferometers.

\section{Acknowledgments}

The authors would like to thank Gareth Jones for his help in the understanding of the LAL parameter system. This work is partially supported by the National Science Foundation grant 
NSF-0838740, a NSF Gravitational Physics grant (PHY-0653321) to VK, NSF Gravitational Physics grant (PHY-0553422) to NC and the Max-Planck-Society (CR). Computations were performed on the Fugu computer cluster funded by NSF MRI grant PHY-0619274 to VK.

\section{References}

Acernese F et al 2004 Class. Quantum Grav. 21 S385-94

Ajith P and Bose S 2009 arXiv:0901.4936

Apostolatos T A, Cutler C, Sussman G J and Thorne K S 1994 Phys. Rev. D 49 6274-97

Atchadé Y F and Rosenthal J S 2005 Bernoulli 11 815-28

Barish B C and Weiss R 1999 Phys. Today 52 44-50

Beauville F et al (The joint LIGO/Virgo working group) 2008 Class. Quantum Grav. 25045001

Blanchet L, Buonanno A and Faye G 2006 Phys. Rev. D 74104034

Buonanno A, Chen Y and Vallisneri M 2003 Phys. Rev. D 67104025

Cavalier F, Barsuglia M, Bizouard M A, Brisson V, Clapson A C, Davier M, Hello P, Kreckelbergh S, Leroy N and Varvella M 2006 Phys. Rev. D 74082004

Cutler C and Thorne K S 2002 Proc. GR16 (Singapore: World Scientific)

Faye G, Blanchet L and Buonanno A 2006 Phys. Rev. D 74104033

Figueiredo M A T and Jain A K 2002 IEEE Trans. Pattern Anal. Mach. Intell. 24 381-96

Fritschel P 2003 Society of Photo-Optical Instrumentation Engineers (SPIE) Conf. Ser. vol 4856 ed M Cruise and P Saulson pp 282-91

Gair J R, Porter E, Babak S and Barack L 2008 Class. Quantum Grav. 25184030

Gelman A, Carlin J B, Stern H and Rubin D B 1997 Bayesian Data Analysis (Boca Raton, FL: Chapman and Hall)

Gilks W R, Richardson S and Spiegelhalter D J 1996 Markov Chain Monte Carlo in Practice (London: Chapman and Hall)

Grandclément P, Kalogera V and Vecchio A 2003 Phys. Rev. D 67042003

Kidder L E 1995 Phys. Rev. D 52 821-47

Lang R N and Hughes S A 2006 Phys. Rev. D 74122001

LIGO Scientific Collaboration 2007 The LSC algorithm library https://www.lsc-group.phys.uwm.edu/daswg/projects/lal.html

Markowitz J, Zanolin M, Cadonati L and Katsavounidis E 2008 Phys. Rev. D 78122003

O’Shaughnessy R, Kim C, Kalogera V and Belczynski K 2008 Astrophys. J. 672 479-88

Röver C, Meyer R and Christensen N 2006 Class. Quantum Grav. 23 4895-906

Röver C, Meyer R and Christensen N 2007a Phys. Rev. D 75062004

Röver C, Meyer R, Guidi G M, Viceré A and Christensen N 2007b Class. Quantum Grav. 24607

Searle A C, Sutton P J and Tinto M 2008 arXiv:0809.2809

Stroeer A et al 2007 Class. Quantum Grav. 24541

van der Sluys M, Raymond V, Mandel I, Röver C, Christensen N, Kalogera V, Meyer R and Vecchio A 2008a Class. Quantum Grav. 25184011

van der Sluys M V, Röver C, Stroeer A, Raymond V, Mandel I, Christensen N, Kalogera V, Meyer R and Vecchio A 2008b Astrophys. J. $\mathbf{6 8 8}$ L61-4

Vecchio A 2004 Phys. Rev. D 70042001

Will C M and Wiseman A G 1996 Phys. Rev. D 54 4813-48

Willke B et al 2004 Class. Quantum Grav. 21417 